\title{
Mathematical Modeling for Hospitalization due to Temperature Variations
}

\author{
Nita H. Shah ${ }^{a^{*}}$, Purvi M. Pandya ${ }^{b}$, Moksha H. Satiac ${ }^{c}$, Foram A. Thakkar \\ Department of Mathematics \\ Gujarat University, Ahmedabad - 380009, Gujarat, India \\ E-mails: ${ }^{a}$ nitahshah@gmail.com, ${ }^{\mathrm{b}}$ pandya091@gmail.com, ${ }^{\mathrm{c}}$ mokshasatia.05@gmail.com, \\ dforamkhakhar3@gmail.com \\ ${ }^{*}$ Corresponding author
}

(Received October 26, 2018; Accepted January 10, 2019)

\begin{abstract}
Sudden changes in temperature occur due to ecological disturbances which results into global warming, volcanic eruption, depletion of ozone layer etc. This has become a crucial environmental and social issue all over the world. Changes in temperature influences transmission pattern for several diseases which leads to increase in the death rate. This research focusses on hospitalization due to temperature variations. Here, class of exposed individuals are divided into two types: asymptomatic individuals who do not show any symptoms and symptomatic individuals which shows symptoms. The indoor or outdoor medications are the remedial steps to get cured. Severe dehydration, blood pressure fluctuations etc. need hospitalization. The rate at which the individual gets hospitalized is scrutinized using SEIRS model. The system of non-linear ordinary differential equations is formulated for the given model and the reproduction number is then computed using next generation matrix method which connotes the recovery rate of an individual. Stability and numerical simulations are carried out.
\end{abstract}

Keywords: Mathematical model, Basic reproduction number, Stability, Simulation.

\section{Introduction}

Temperature is a measure of the warmth or coldness of an object or substance with reference to some standard value. In the proposed model, temperature refers to ambient temperature. Change in temperature can occur at any location for any period. On this basis, it is classified as in Figure 1.

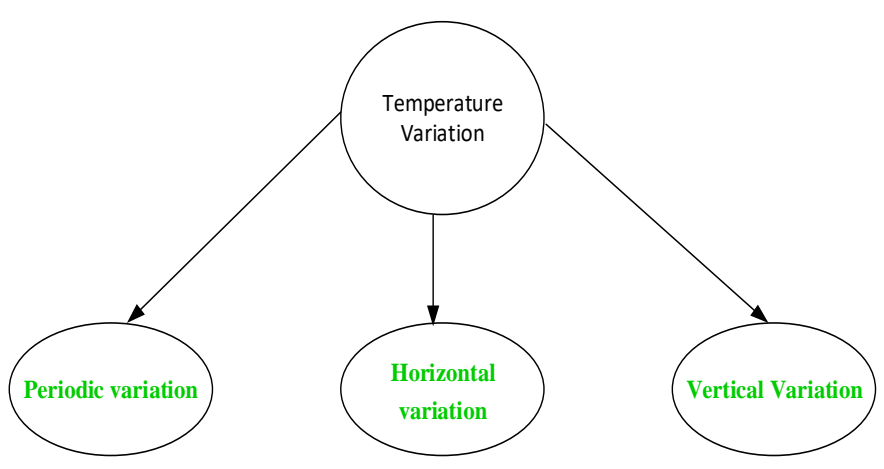

Figure 1. Classification of temperature variation

Periodic temperature variation is the continuous change of temperature during a day, week, month, year or any period, whereas a horizontal temperature variation is the change of solar energy with 
International Journal of Mathematical, Engineering and Management Sciences

Vol. 4, No. 2, 361-374, 2019

https://dx.doi.org/10.33889/IJMEMS.2019.4.2-029

respect to equatorial region and latitude. Vertical temperature variation is divided according to four spheres namely troposphere, stratosphere, mesosphere and thermosphere. This model is based on periodic temperature variations. During the last few years, the association between extreme temperatures and health issues has been frequently investigated. Unstable changes in temperature disturbs the weather we experience and our basic necessities resulting in increased health issues. The sternness of these health risks (hospitalization rate) depends on factors such as age, gender, individual's strength to sustain temperature change and monetary status. It will also vary based on location, sensitivity and adapting capability of an individual. Health effects may include gastrointestinal illness like vomiting, diarrhoea, effects on the body's nervous and respiratory systems, liver and kidney damage, etc. Some health issues may show some symptoms, others may not. Those individuals who show symptoms such as dehydration, low blood pressure, respiratory problems, heat stroke etc. are known as symptomatic individuals and those who do not show any symptoms such as any type of infection are asymptomatic individuals. Proper intime treatment is required to control these health issues. If the condition becomes critical and cannot be controlled by taking indoor medications, they get hospitalized. This research is focused on transmission rate of any health issue due to temperature variations on an individual from susceptible stage to hospitalised stage.

A lot of survey is done about effects of climate change on human health such as in book "Global climate change and health: an old story writ large" (McMichael, 2003) which contains information about every issue related to climate change and human health: Risks and responses. It is also reviewed (Wu et al., 2016) about the empirical evidence and human adaptation due to climate change on human infectious diseases. One of the study (Paaijmans et al., 2010) showed the influence of climate on malaria transmission which depends on daily temperature variation. The impact of rainfall and temperature variation on diarrheal prevalence in sub-saharan Africa was studied (Bandyopadhyay et al., 2012). A dynamic model of air pollution, health and population growth was examined using system dynamics: a study on Tehran-Iran (Shahgholian and Hajihosseini, 2009). Direct and indirect effect of climate change was analysed on human health (McMichael et al., 1997). Analysis was done for greater temperature variation within a day associated with increased emergency hospital admissions for asthma (Qiu et al., 2015). The temperature variation and emergency hospital admissions were reported for stroke in Brisbane, Australia, 1996-2005 (Wang et al., 2009). The impact was studied Zell et al. (2008) for global warming on viral diseases. Also, the basics of SEIR model were discussed and applied it for vector borne diseases to analyse steady state conditions. Also, after finding threshold parameter $R_{0}$ it was shown that the disease will spread only if its value exceeds 1 (Shah and Gupta, 2013, 2014). A mathematical model was formulated for water pollutants with stability analysis (Shah et al., 2018). A preliminary mathematical model was also computed for the transmission of dengue, chikungunya and zika (Isea and Lonngren, 2016). Similarly, a system of ordinary differential equations was designed to simulate the transmission of infection between humans and Aedes albopictus (Yakob and Clements, 2013).

The mathematical model for hospitalization rate is shown in Section 2 followed by stability at equilibrium points in Section 3. Numerical simulation is then computed in Section 4, concluding the model finally in Section 5.

\section{Mathematical Model}

The hospitalization rate due to temperature variations is studied in this mathematical model. It is based on SEIRS-model. This model consists of five compartments, namely, susceptible individual 
International Journal of Mathematical, Engineering and Management Sciences

Vol. 4, No. 2, 361-374, 2019

https://dx.doi.org/10.33889/IJMEMS.2019.4.2-029

$\left(S_{T}\right)$ who are likely to be influenced by temperature variations which are then exposed $(E)$ to temperature variations. These individuals are then classified into two: those who do not symptoms i.e. asymptomatic $(A)$ and who show symptoms which are symptomatic $(S)$ individuals. Symptomatic individuals after taking some medications may become asymptomatic and vice-versa. If the rate of asymptomatic and symptomatic individuals cannot be controlled by taking indoor medications, they get hospitalized $(H)$. After getting cured, they may again get susceptible.

Table 1. Notations and parametric values

\begin{tabular}{|c|l|c|}
\hline Notation & \multicolumn{1}{|c|}{ Description } & $\begin{array}{c}\text { Parametric } \\
\text { values }\end{array}$ \\
\hline$B$ & New recruitment rate & 0.6 \\
\hline$\alpha_{1}$ & The rate at which susceptible individuals gets exposed & 0.5 \\
\hline$\alpha_{2}$ & The rate at which exposed individuals are asymptomatic & 0.01 \\
\hline$\alpha_{3}$ & The rate at which exposed individuals start showing symptoms & 0.02 \\
\hline$\alpha_{4}$ & The rate at which asymptomatic individuals becomes symptomatic & 0.12 \\
\hline$\alpha_{5}$ & $\begin{array}{l}\text { The rate at which symptomatic individuals after taking proper medications } \\
\text { asymptomatic }\end{array}$ & 0.05 \\
\hline$\alpha_{6}$ & The rate at which asymptomatic individuals gets hospitalized & 0.02 \\
\hline$\alpha_{7}$ & The rate at which symptomatic individuals is hospitalized & 0.05 \\
\hline$\alpha_{8}$ & The rate at which hospitalized individuals after getting cured again becomes susceptible & 0.08 \\
\hline$\mu$ & Mortality rate & 0.03 \\
\hline
\end{tabular}

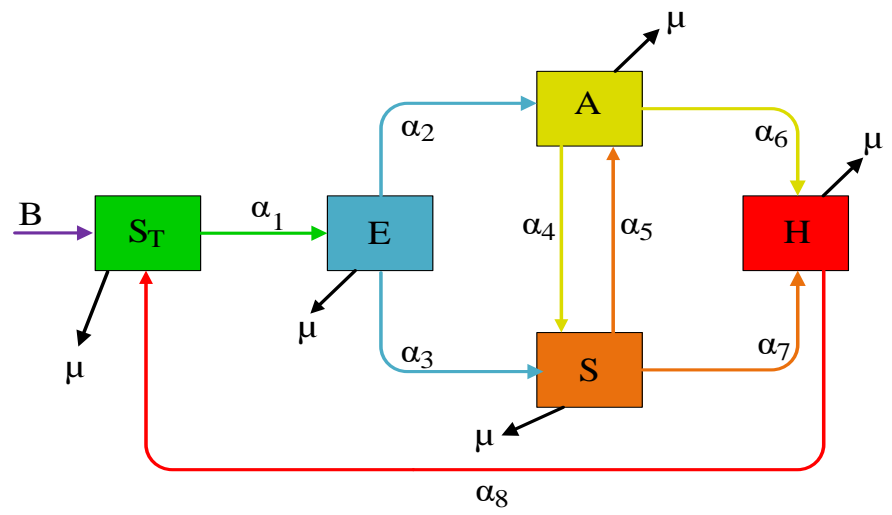

Figure 2. Transmission diagram for hospitalization

Using parametric values, the mathematical model shown in Figure 2 is formulated. The system of non-linear differential equations is as follows: 
International Journal of Mathematical, Engineering and Management Sciences

Vol. 4, No. 2, 361-374, 2019

https://dx.doi.org/10.33889/IJMEMS.2019.4.2-029

$\frac{d S_{T}}{d t}=B-\alpha_{1} S_{T} E+\alpha_{8} H-\mu S_{T}$

$\frac{d E}{d t}=\alpha_{1} S_{T} E-\alpha_{2} E A-\alpha_{3} E S-\mu E$

$\frac{d A}{d t}=\alpha_{2} E A+\alpha_{5} S-\alpha_{4} A-\alpha_{6} A-\mu A$

$\frac{d S}{d t}=\alpha_{3} E S+\alpha_{4} A-\alpha_{5} S-\alpha_{7} S-\mu S$

$\frac{d H}{d t}=\alpha_{6} A+\alpha_{7} S-\alpha_{8} H-\mu H$

where, $S_{T}+E+A+S+H \leq N$. Also, $S_{T}>0 ; \mathrm{E}, \mathrm{A}, S, \mathrm{H} \geq 0$.

Adding the above differential equations of system (1), we have

$\frac{d}{d t}\left(S_{T}+E+A+S+H\right)=B-\mu\left(S_{T}+E+A+S+H\right) \geq 0 ; \quad$ which $\quad$ implies that $\limsup _{t \rightarrow \infty}\left(S_{T}+E+A+S+H\right) \leq \frac{B}{\mu}$.

Therefore, the feasible region of the model is $\Lambda=\left\{\left(S_{T}, E, A, S, H\right) \in R^{5}: S_{T}+E+A+S+H \leq \frac{B}{\mu}\right\}$.

Now, the disease-free equilibrium point of the transmission of model is $Y_{0}=\left(\frac{B}{\mu}, 0,0,0,0\right)$.

Unaffected equilibrium point is $Y_{1}=\left(\frac{\mu}{\alpha_{1}}, \frac{B \alpha_{1}-\mu^{2}}{\alpha_{1} \mu}, 0,0,0\right)$ and endemic point is $Y^{*}=\left(S_{T}^{*}, E^{*}, A^{*}, S^{*}, H^{*}\right)$

where $S_{T}^{*}=\frac{\left(r \alpha_{3}-\alpha_{5}-\alpha_{7}-\mu\right)\left(B \alpha_{2}\left(\alpha_{8}+\mu\right)-\alpha_{6} \alpha_{8} \mu\right)-\alpha_{4}\left(B \alpha_{3}\left(\alpha_{8}+\mu\right)+\alpha_{7} \alpha_{8} \mu\right)}{\mu\left[\begin{array}{r}\alpha_{1}\left\{\left(\alpha_{6}+\alpha_{8}+\mu\right)\left(\alpha_{3} r-\alpha_{5}\right)-\alpha_{4}\left(\alpha_{7}+\alpha_{8}+\mu\right)-\alpha_{6}\left(\alpha_{7}+\mu\right)\right\} \\ +\left(\alpha_{8}+\mu\right)\left(\alpha_{2}\left(\alpha_{3} r-\alpha_{5}\right)-\left(\alpha_{7}+\mu\right)\left(\alpha_{1}+\alpha_{2}\right)-\alpha_{3} \alpha_{4}\right)\end{array}\right]}$,

$E^{*}=r$,

$A^{*}=\frac{\left(\alpha_{2}\left(r \alpha_{3}-\alpha_{5}-\alpha_{7}-\mu\right)+\alpha_{3} \alpha_{5}\right)\left(\alpha_{1}(B-r \mu)-\mu^{2}\right)\left(\alpha_{8}+\mu\right)}{\mu\left[\begin{array}{c}r \alpha_{2} \alpha_{3}\left(\alpha_{1}\left(\alpha_{6}-\alpha_{7}\right)+\left(\alpha_{8}+\mu\right)\left(\alpha_{2}-\alpha_{3}\right)\right) \\ -\alpha_{1} \alpha_{2}\left(\left(\alpha_{6}+\alpha_{8}+\mu\right)\left(\alpha_{5}+\alpha_{7}+\mu\right)+\alpha_{4}\left(\alpha_{7}+\alpha_{8}+\mu\right)\right) \\ +\alpha_{1} \alpha_{3}\left(\left(\alpha_{7}+\alpha_{8}+\mu\right)\left(\alpha_{4}+\alpha_{6}+\mu\right)+\alpha_{5}\left(\alpha_{6}+\alpha_{8}+\mu\right)\right) \\ -\left(\alpha_{8}+\mu\right)\left(\alpha_{2}^{2}\left(\alpha_{5}+\alpha_{7}+\mu\right)+\alpha_{2} \alpha_{3}\left(\alpha_{4}-\alpha_{5}\right)-\alpha_{3}^{2}\left(\alpha_{4}+\alpha_{6}+\mu\right)\right)\end{array}\right]}$, 
International Journal of Mathematical, Engineering and Management Sciences

Vol. 4, No. 2, 361-374, 2019

https://dx.doi.org/10.33889/IJMEMS.2019.4.2-029

$$
\begin{aligned}
S^{*}= & \frac{\alpha_{4}\left(\alpha_{8}+\mu\right)\left(\mathrm{r} \alpha_{1} \mu-\mathrm{B} \alpha_{1}+\mu^{2}\right)}{\mu\left[\begin{array}{r}
\alpha_{1}\left\{\left(\alpha_{6}+\alpha_{8}+\mu\right)\left(\alpha_{3} r-\alpha_{5}\right)-\alpha_{4}\left(\alpha_{7}+\alpha_{8}+\mu\right)-\alpha_{6}\left(\alpha_{7}+\mu\right)\right\} \\
+\left(\alpha_{8}+\mu\right)\left(\alpha_{2}\left(r \alpha_{3}-\alpha_{5}\right)-\left(\alpha_{1}+\alpha_{2}\right)\left(\alpha_{7}+\mu\right)-\alpha_{3} \alpha_{4}\right)
\end{array}\right]}, \\
H^{*}= & \frac{+\alpha_{6} \mu\left(\alpha_{1}\left(\alpha_{6}+\mu\right)\left(\alpha_{5}+\alpha_{7}+\mu\right)+\left(\alpha_{7}+\mu\right)\left(\alpha_{1} \alpha_{4}+\alpha_{2} \mu\right)\right)+\alpha_{2} \mu^{2}\left(\alpha_{4} \alpha_{7}+\alpha_{5} \alpha_{6}\right)}{\alpha_{1} \alpha_{2}\left(\alpha_{6}\left(r \alpha_{3}-\alpha_{5}-\alpha_{7}-\mu\right)-\alpha_{4} \alpha_{7}\right)+r \mu\left(\alpha_{1}\left(\alpha_{2} \alpha_{4} \alpha_{7}-\alpha_{3} \alpha_{6}\left(\alpha_{4}+\alpha_{6}+\mu\right)\right)-\alpha_{2} \alpha_{3} \alpha_{6} \mu\right)} \\
& \alpha_{2} \mu\left[\begin{array}{c}
\alpha_{1}\left\{\left(\alpha_{6}+\alpha_{8}+\mu\right)\left(\alpha_{3} r-\alpha_{5}\right)-\alpha_{4}\left(\alpha_{7}+\alpha_{8}+\mu\right)-\alpha_{6}\left(\alpha_{7}+\mu\right)\right\} \\
+\left(\alpha_{8}+\mu\right)\left(r \alpha_{3}\left(\alpha_{3}-\alpha_{4}\right)-\left(\alpha_{7}+\mu\right)\left(\alpha_{1}+\alpha_{2}\right)-\alpha_{2} \alpha_{5}\right)
\end{array}\right]
\end{aligned}
$$

and

$$
r=\frac{1}{2 \alpha_{2} \alpha_{3}}\left[\begin{array}{c}
\alpha_{2}\left(\alpha_{5}+\alpha_{7}+\mu\right)+\alpha_{3}\left(\alpha_{4}+\alpha_{6}+\mu\right) \\
+\left\{\begin{array}{c}
\alpha_{2}^{2}\left(\alpha_{5}\left(\alpha_{5}+2 \alpha_{7}+2 \mu\right)+\left(\alpha_{7}+\mu\right)^{2}\right) \\
+2 \alpha_{2} \alpha_{3}\left(\alpha_{4}\left(\alpha_{5}-\alpha_{7}-\mu\right)-\left(\alpha_{6}+\mu\right)\left(\alpha_{5}+\alpha_{7}+\mu\right)\right) \\
+\alpha_{3}^{2}\left(\alpha_{4}\left(\alpha_{4}+2 \alpha_{6}+2 \mu\right)+\left(\alpha_{6}+\mu\right)^{2}\right)
\end{array}\right\}^{\frac{1}{2}}
\end{array}\right] .
$$

Now, we calculate the threshold called basic reproduction number $R_{0}$ using next generation matrix method (Diekmann et al., 2009).

Let $X^{\prime}=\left(S_{T}, E, A, S, H\right)^{\prime}$ and $X^{\prime}=\frac{d X}{d t}=F(X)-V(X)$, where $F(X)$ denotes the rate of appearance of new individual in the compartment and $V(X)$ denotes the rate of transfer of health issue which are given by

$$
F(X)=\left[\begin{array}{c}
\alpha_{1} S_{T} E \\
\alpha_{2} E A \\
\alpha_{3} E S \\
0 \\
0
\end{array}\right] \text { and } V(X)=\left[\begin{array}{c}
E\left(\alpha_{2} A+\alpha_{3} S+\mu\right) \\
-\alpha_{5} S+A\left(\alpha_{4}+\alpha_{6}+\mu\right) \\
-\alpha_{4} A+S\left(\alpha_{5}+\alpha_{7}+\mu\right) \\
-\alpha_{6} A-\alpha_{7} S+H\left(\alpha_{8}+\mu\right) \\
-B+\alpha_{1} S_{T} E-\alpha_{8} H+\mu S_{T}
\end{array}\right]
$$

Now, $D F\left(X_{0}\right)=\left[\begin{array}{cc}f & 0 \\ 0 & 0\end{array}\right]$ and $D V\left(X_{0}\right)=\left[\begin{array}{cc}v & 0 \\ J_{1} & J_{2}\end{array}\right]$, where $f$ and $v$ are $5 \times 5$ matrices defined as

$$
f=\left[\frac{\partial F_{i}\left(\mathrm{X}_{0}\right)}{\partial \mathrm{X}_{j}}\right] \text { and } v=\left[\frac{\partial V_{i}\left(\mathrm{X}_{0}\right)}{\partial \mathrm{X}_{j}}\right]
$$

Finding $f$ and $v$, we get 
International Journal of Mathematical, Engineering and Management Sciences

Vol. 4, No. 2, 361-374, 2019

https://dx.doi.org/10.33889/IJMEMS.2019.4.2-029

$$
f=\left[\begin{array}{ccccc}
\alpha_{1} S_{T} & 0 & 0 & 0 & \alpha_{1} E \\
\alpha_{2} A & \alpha_{2} E & 0 & 0 & 0 \\
\alpha_{3} S & 0 & \alpha_{3} E & 0 & 0 \\
0 & 0 & 0 & 0 & 0 \\
0 & 0 & 0 & 0 & 0
\end{array}\right] \text { and } v=\left[\begin{array}{ccccc}
\alpha_{2} A+\alpha_{3} S+\mu & \alpha_{2} E & \alpha_{3} E & 0 & 0 \\
0 & \alpha_{4}+\alpha_{6}+\mu & -\alpha_{5} & 0 & 0 \\
0 & -\alpha_{4} & \alpha_{5}+\alpha_{7}+\mu & 0 & 0 \\
0 & -\alpha_{6} & -\alpha_{7} & \alpha_{8}+\mu & 0 \\
\alpha_{1} S_{T} & 0 & 0 & -\alpha_{8} & \alpha_{1} E+\mu
\end{array}\right] .
$$

Here, $v$ is non-singular matrix.

For this model, the basic reproduction number $R_{0}$ is not permissible at $Y_{0}$. So, we find $R_{0}$ at $Y_{1}=\left(\frac{\mu}{\alpha_{1}}, \frac{B \alpha_{1}-\mu^{2}}{\alpha_{1} \mu}, 0,0,0\right)$ which is obtained as the spectral radius of matrix $f v^{-1}$.

$$
R_{0}=\frac{\left(\begin{array}{c}
\alpha_{2} \mu^{3}+\left(\alpha_{2}\left\{\alpha_{5}+\alpha_{7}+\alpha_{8}\right\}+\alpha_{3} \alpha_{4}\right) \mu^{2}-\alpha_{8}\left(\alpha_{1} \alpha_{6}-\alpha_{2}\left\{\alpha_{5}-\alpha_{7}\right\}-\alpha_{3} \alpha_{4}\right) \mu \\
-\alpha_{8} \alpha_{1}\left(\left\{\alpha_{4}+\alpha_{6}\right\} \alpha_{7}+\alpha_{5} \alpha_{6}\right)
\end{array}\right)\left(\mu^{2}-B \alpha_{1}\right)}{\alpha_{1}^{2}\left(\mu^{2}+\left(\alpha_{4}+\alpha_{5}+\alpha_{6}+\alpha_{7}\right) \mu+\left(\alpha_{4}+\alpha_{6}\right) \alpha_{7}+\alpha_{5} \alpha_{6}\right) B\left(\alpha_{8}+\mu\right)}
$$

\section{Stability}

In this section, for all equilibrium points local as well as global stability is discussed.

\subsection{Local Stability}

Theorem 3.1.1 (Stability at $\left.Y_{0}\right)$ : If $\alpha_{1} B<\mu^{2}$ then $Y_{0}=\left(\frac{B}{\mu}, 0,0,0,0\right)$ is locally asymptotically stable.

Proof: The Jacobian matrix $J_{0}$ at $Y_{0}$ is given by

$$
J_{0}=\left[\begin{array}{ccccc}
-\mu & -\frac{\alpha_{1} B}{\mu} & 0 & 0 & 0 \\
0 & \frac{\alpha_{1} B}{\mu}-\mu & 0 & 0 & 0 \\
0 & 0 & -\left(\alpha_{4}+\alpha_{6}+\mu\right) & \alpha_{5} & 0 \\
0 & 0 & \alpha_{4} & -\left(\alpha_{5}+\alpha_{7}+\mu\right) & 0 \\
0 & 0 & \alpha_{6} & \alpha_{7} & -\left(\alpha_{8}+\mu\right)
\end{array}\right] .
$$

Above Jacobian $J_{0}$ has eigenvalues,

$$
\begin{aligned}
& \lambda_{1}=-\mu<0, \lambda_{2}=-\left(\alpha_{8}+\mu\right)<0, \\
& \lambda_{3}=-\left(\frac{\left(\alpha_{4}+\alpha_{5}+\alpha_{6}+\alpha_{7}+2 \mu\right)}{2}-\frac{\sqrt{\left(\alpha_{4}+\alpha_{5}\right)^{2}+\left(\alpha_{6}-\alpha_{7}\right)^{2}+2\left(\alpha_{4}-\alpha_{5}\right)\left(\alpha_{6}-\alpha_{7}\right)}}{2}\right)<0,
\end{aligned}
$$


International Journal of Mathematical, Engineering and Management Sciences

Vol. 4, No. 2, 361-374, 2019

https://dx.doi.org/10.33889/IJMEMS.2019.4.2-029

$$
\begin{aligned}
& \lambda_{4}=-\left(\frac{\left(\alpha_{4}+\alpha_{5}+\alpha_{6}+\alpha_{7}+2 \mu\right)}{2}+\frac{\sqrt{\left(\alpha_{4}+\alpha_{5}\right)^{2}+\left(\alpha_{6}-\alpha_{7}\right)^{2}+2\left(\alpha_{4}-\alpha_{5}\right)\left(\alpha_{6}-\alpha_{7}\right)}}{2}\right)<0, \\
& \lambda_{5}=\frac{\alpha_{1} B-\mu^{2}}{\mu}<0 \text { if } \alpha_{1} B<\mu^{2} .
\end{aligned}
$$

As all eigenvalues of above matrix are negative. Therefore, equilibrium point of system is stable.

Theorem 3.1.2 (Stability at $Y_{1}$ ): If $x_{2} x_{3}<\alpha_{4} \alpha_{5}$ where $x_{1}=\frac{B \alpha_{1}-\mu^{2}}{\mu}, x_{2}=\frac{\alpha_{2} x_{1}}{\alpha_{1}}-\alpha_{4}-\alpha_{6}-\mu$ and $x_{3}=\frac{\alpha_{3} x_{1}}{\alpha_{1}}-\alpha_{5}-\alpha_{7}-\mu$ then $Y_{1}=\left(\frac{\mu}{\alpha_{1}}, \frac{B \alpha_{1}-\mu^{2}}{\alpha_{1} \mu}, 0,0,0\right)$ is locally asymptotically stable.

Proof: For $Y_{1}=\left(\frac{\mu}{\alpha_{1}}, \frac{B \alpha_{1}-\mu^{2}}{\alpha_{1} \mu}, 0,0,0\right)$, let $\frac{B \alpha_{1}-\mu^{2}}{\mu}=x_{1}$. The Jacobian matrix $J_{1}$ is given by $J_{1}=\left[\begin{array}{ccccc}-\left(\mu+x_{1}\right) & -\mu & 0 & 0 & 0 \\ x_{1} & 0 & -\frac{\alpha_{2} x_{1}}{\alpha_{1}} & -\frac{\alpha_{3} x_{1}}{\alpha_{1}} & 0 \\ 0 & 0 & \frac{\alpha_{2} x_{1}}{\alpha_{1}}-\alpha_{4}-\alpha_{6}-\mu & \alpha_{5} & 0 \\ 0 & 0 & \alpha_{4} & \frac{\alpha_{3} x_{1}}{\alpha_{1}}-\alpha_{5}-\alpha_{7}-\mu & 0 \\ 0 & 0 & \alpha_{6} & \alpha_{7} & -\left(\alpha_{8}+\mu\right)\end{array}\right]$.

Above Jacobian $J_{1}$ has eigenvalues

$$
\begin{aligned}
& \lambda_{1}=-x_{1}<0, \lambda_{2}=-\mu<0, \lambda_{3}=-\left(\alpha_{8}+\mu\right)<0 \\
& \lambda_{4}=\frac{x_{2}+x_{3}}{2}+\frac{\sqrt{4 \alpha_{4} \alpha_{5}+\left(x_{2}-x_{3}\right)^{2}}}{2}<0 \text { if } x_{2} x_{3}<\alpha_{4} \alpha_{5}, \lambda_{5}=\frac{x_{2}+x_{3}}{2}-\frac{\sqrt{4 \alpha_{4} \alpha_{5}+\left(x_{2}-x_{3}\right)^{2}}}{2}<0 .
\end{aligned}
$$

Here, all the eigen values are negative. So, $Y_{1}$ is locally asymptomatically stable.

Theorem 3.1.3 (Stability at $Y^{*}$ ): Endemic point $Y^{*}$ is locally asymptotically stable if the following conditions hold:

i) $x_{6} x_{7}>\alpha_{4} \alpha_{5}$, ii) $\alpha_{2} A^{*}+\alpha_{3} S^{*}+\mu>\alpha_{1} S_{T}$, iii) $\alpha_{4}+\alpha_{6}+\mu>\alpha_{2} E^{*}$, iv) $\alpha_{5}+\alpha_{7}+\mu>\alpha_{3} E^{*}$

Proof: We determine the local stability behavior of endemic equilibrium point $Y^{*}$ by using the Jacobian matrix $J^{*}$, which is given by 
International Journal of Mathematical, Engineering and Management Sciences

Vol. 4, No. 2, 361-374, 2019

https://dx.doi.org/10.33889/IJMEMS.2019.4.2-029

$$
J^{*}=\left[\begin{array}{ccccc}
-\left(\alpha_{1} E^{*}+\mu\right) & -\alpha_{1} S_{T}^{*} & 0 & 0 & 0 \\
\alpha_{1} E^{*} & \alpha_{1} S_{T}^{*}-\alpha_{2} A^{*}-\alpha_{3} S^{*}-\mu & -\alpha_{2} E^{*} & -\alpha_{3} E^{*} & 0 \\
0 & \alpha_{2} A^{*} & \alpha_{2} E^{*}-\alpha_{4}-\alpha_{6}-\mu & \alpha_{5} & 0 \\
0 & \alpha_{3} S^{*} & \alpha_{4} & \alpha_{3} E^{*}-\alpha_{5}-\alpha_{7}-\mu & 0 \\
0 & 0 & \alpha_{6} & \alpha_{7} & -\left(\alpha_{8}+\mu\right)
\end{array}\right] .
$$

Let,

$x_{4}=\alpha_{1} E^{*}+\mu, x_{5}=\alpha_{2} A^{*}+\alpha_{3} S^{*}-\alpha_{1} S_{T}^{*}+\mu, x_{6}=\alpha_{4}+\alpha_{6}-\alpha_{2} E^{*}+\mu, x_{7}=\alpha_{5}+\alpha_{7}-\alpha_{3} E^{*}+\mu$, $x_{8}=\alpha_{8}+\mu$.

The corresponding characteristic equation of Jacobian matrix is

$\lambda^{5}+a_{1} \lambda^{4}+a_{2} \lambda^{3}+a_{3} \lambda^{2}+a_{4} \lambda+a_{5}=0$ where

$$
\begin{aligned}
& a_{1}=x_{4}+x_{5}+x_{6}+x_{7}+x_{8} \\
& a_{2}=E^{*}\left(\alpha_{2}^{2} A^{*}+\alpha_{3}^{2} S^{*}+\alpha_{1}^{2} S_{T}^{*}\right)+\left(x_{6} x_{7}-\alpha_{4} \alpha_{5}\right)+\left(x_{4}+x_{5}\right)\left(x_{6}+x_{7}+x_{8}\right)+x_{8}\left(x_{6}+x_{7}\right)+x_{4} x_{5} \\
& a_{3}=E^{*}\left(\alpha_{2}^{2} A^{*}\left(x_{4}+x_{7}+x_{8}\right)+\alpha_{2} \alpha_{3}\left(A^{*} \alpha_{4}+S^{*} \alpha_{5}\right)+\alpha_{3}^{2} S^{*}\left(x_{4}+x_{6}+x_{8}\right)\right) \\
& +\left(x_{6}+x_{7}+x_{8}\right)\left(E^{*} S_{T}^{*} \alpha_{1}^{2}+x_{4} x_{5}\right)+\left(x_{6} x_{7}-\alpha_{4} \alpha_{5}\right)\left(x_{4}+x_{5}+x_{8}\right)+\left(x_{4}+x_{5}\right)\left(x_{6}+x_{7}\right) x_{8} \\
& a_{4}=A^{*} E^{*} \alpha_{2}\left(\alpha_{2} x_{4}\left(x_{7}+x_{8}\right)+x_{7}\left(\alpha_{2} x_{8}+\alpha_{3} x_{4}\right)+\alpha_{3}\left(x_{7} x_{8}+\alpha_{5}\left(x_{4}+x_{8}\right)\right)\right) \\
& +E^{*} S^{*} \alpha_{3}^{2}\left(x_{4} x_{8}+x_{6}\left(x_{4}+x_{8}\right)\right)+\left(x_{6} x_{7}-\alpha_{4} \alpha_{5}\right)\left(E^{*} S_{T}^{*} \alpha_{1}^{2}+x_{4} x_{8}+x_{5}\left(x_{4}+x_{8}\right)\right) \\
& +\left(x_{6}+x_{7}\right) x_{8}\left(E^{*} S_{T}^{*} \alpha_{1}^{2}+x_{4} x_{5}\right) \\
& a_{5}=x_{8}\left(E^{*} x_{4}\left(A^{*} \alpha_{2}\left(\alpha_{2} x_{7}+\alpha_{3} \alpha_{4}\right)+S^{*} \alpha_{3}\left(\alpha_{2} \alpha_{5}+\alpha_{3} x_{6}\right)\right)+\left(x_{6} x_{7}-\alpha_{4} \alpha_{5}\right)\left(E^{*} S_{T}^{*} \alpha_{1}^{2}+x_{4} x_{5}\right)\right) \text {. }
\end{aligned}
$$

The equilibrium point is locally asymptotically stable (Routh, 1877), if following conditions are hold:

i) $x_{6} x_{7}>\alpha_{4} \alpha_{5}$, ii) $\alpha_{2} A^{*}+\alpha_{3} S^{*}+\mu>\alpha_{1} S_{T}$, iii) $\alpha_{4}+\alpha_{6}+\mu>\alpha_{2} E^{*}$, iv) $\alpha_{5}+\alpha_{7}+\mu>\alpha_{3} E^{*}$.

\subsection{Global Stability}

Here, we discuss the global stability behavior of the equilibrium $Y_{0}, Y_{1}$ and $Y^{*}$ by Lyapunov's function.

Theorem 3.2.1 (Stability at $Y_{0}$ ): The disease-free equilibrium point $Y_{0}$ is globally asymptotically stable. 
International Journal of Mathematical, Engineering and Management Sciences

Vol. 4, No. 2, 361-374, 2019

https://dx.doi.org/10.33889/IJMEMS.2019.4.2-029

Proof: Consider the Lyapunov's function $L_{1}(t)=E(t)+A(t)+S(t)+\mathrm{H}(t) \quad$ then $L_{1}^{\prime}(t)=E^{\prime}(t)+A^{\prime}(t)+S^{\prime}(t)+\mathrm{H}^{\prime}(t)=E\left(\alpha_{1} S_{T}-\mu\right)-\alpha_{8} H-\mu(A+S+H)$, we get $\frac{d L}{d t}<0$ if $\alpha_{1} B<\mu^{2}$ whereas $\frac{d L}{d t}=0$ only if $E, A, S, H=0$.

Therefore, $Y_{0}$ is globally asymptotically stable (LaSalle, 1976).

Theorem 3.2.2 (Stability at $Y_{1}$ ): The unaffected equilibrium point $Y_{1}$ is globally asymptotically stable.

Proof: Consider the Lyapunov function $L_{2}(t)=A(t)+S(t)+\mathrm{H}(t)$, then $L_{2}{ }^{\prime}(t)=A^{\prime}(t)+S^{\prime}(t)+\mathrm{H}^{\prime}(t)$ $=A\left(\alpha_{2} E-\mu\right)+S\left(\alpha_{3} E-\mu\right)-H\left(\alpha_{8}+\mu\right)$, we get $\quad \frac{d L}{d t}<0$ if $\quad B \alpha_{1} \alpha_{2}<\mu^{2}\left(\alpha_{1}+\alpha_{2}\right) \quad$ and $B \alpha_{1} \alpha_{3}<\mu^{2}\left(\alpha_{1}+\alpha_{3}\right)$ whereas $\frac{d L}{d t}=0$ only if $A, S, H=0$.

Therefore, $Y_{1}$ is globally asymptotically stable (LaSalle, 1976).

Theorem 3.2.3 (Stability at $Y^{*}$ ): The endemic equilibrium point $Y^{*}$ is globally asymptotically stable.

Proof: Consider the Lyapunov function,

$$
\begin{aligned}
& L(t)=\frac{1}{2}\left[\left(S_{T}-S_{T}^{*}\right)+\left(E-E^{*}\right)+\left(A-A^{*}\right)+\left(S-S^{*}\right)+\left(H-H^{*}\right)\right]^{2}, \text { then } \\
& \begin{array}{c}
L^{\prime}(t)=\left[\left(S_{T}-S_{T}^{*}\right)+\left(E-E^{*}\right)+\left(A-A^{*}\right)+\left(S-S^{*}\right)+\left(H-H^{*}\right)\right]\left(S_{T}{ }^{\prime}+E^{\prime}+A^{\prime}+S^{\prime}+\mathrm{H}^{\prime}\right) \\
=-\mu\left[\left(S_{T}-S_{T}{ }^{*}\right)+\left(E-E^{*}\right)+\left(A-A^{*}\right)+\left(S-S^{*}\right)+\left(H-H^{*}\right)\right]^{2} \leq 0 .
\end{array}
\end{aligned}
$$

Here, we denote $B=\mu\left(S_{T}{ }^{*}+E^{*}+A^{*}+S^{*}+H^{*}\right)$. Therefore, $Y^{*}$ is globally stable. Next, we validate the model by numerical simulation.

\section{Numerical Simulation}

In Figure 3, transmission pattern of humans are observed which are affected due to temperature variations. One can see that if any individual experience sudden temperature change while traveling from one place to another, they gets exposed immediately. Some individuals do not show symptoms for two days and after approximately nine days they begins indicating symptoms. It can also be observed that after approximately eight days six individuals again gets susceptible out of which two individual gets exposed. 
International Journal of Mathematical, Engineering and Management Sciences

Vol. 4, No. 2, 361-374, 2019

https://dx.doi.org/10.33889/IJMEMS.2019.4.2-029

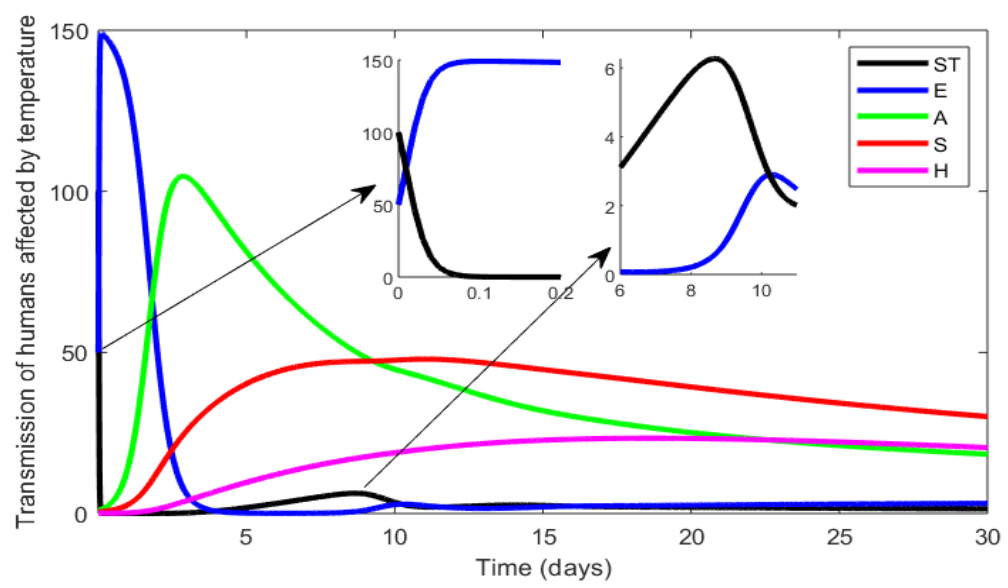

Figure 3. Transmission of humans affected by temperature variations

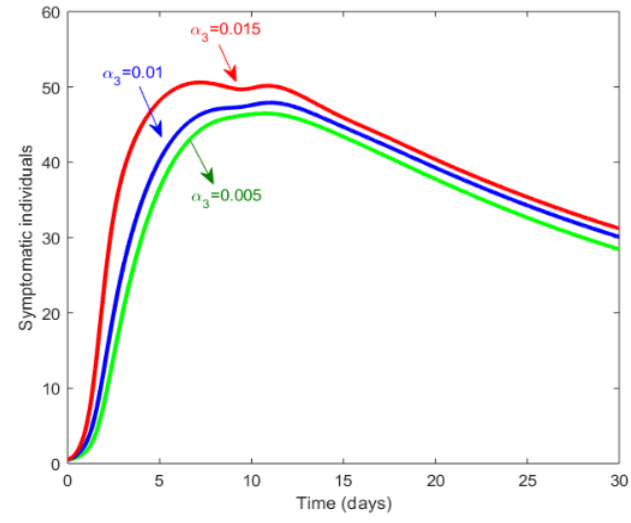

Figure 4. Effect of the rate at which exposed individuals moves to symptomatic individuals

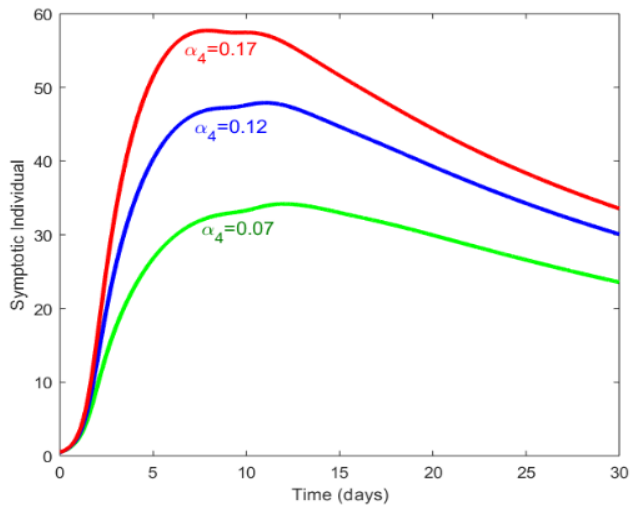

Figure 5. Effect of the rate at which asymptomatic individuals moves to symptomatic individuals 
International Journal of Mathematical, Engineering and Management Sciences

Vol. 4, No. 2, 361-374, 2019

https://dx.doi.org/10.33889/IJMEMS.2019.4.2-029

From Figure 4 and 5, one can observe that increase in rate results in increase of symptomatic individuals. Figure 4 shows that increase in $\alpha_{3}$ by $1 \%$ results in increase of symptomatic individuals by $8 \%$. Figure 5 implies that $64 \%$ increase of symptomatic individuals will be observed if there is an increase in rate of $\alpha_{4}$ by $10 \%$. It also connotes that during exposed or asymptomatic stage if proper precautions or medication are not taken then increase in symptomatic individuals will be observed.

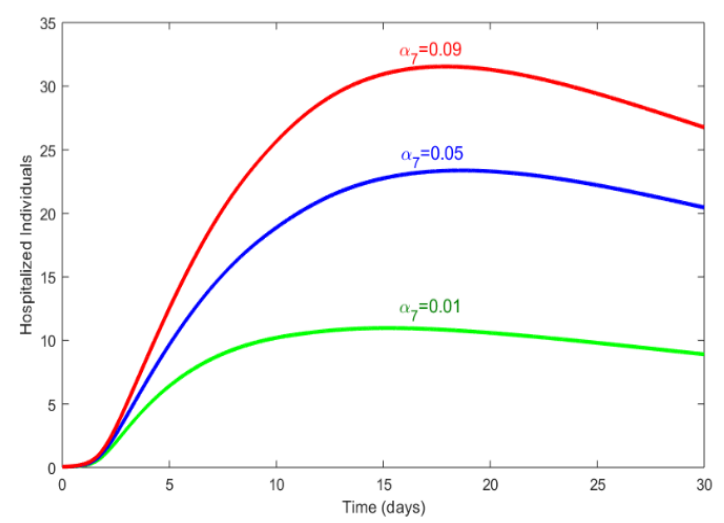

Figure 6. Effect of the rate at which symptomatic individuals moves to hospitalization

Figure 6 shows that $8 \%$ increase in rate of symptomatic individuals results in increase of hospitalized rate from 8 individuals to 26 individuals. So, increase in the rate of symptomatic individuals is directly proportional to hospitalization.

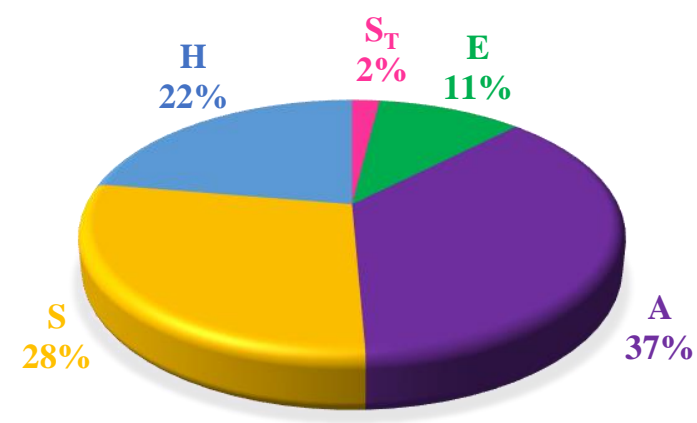

Figure 7. Percentage of all compartments 
International Journal of Mathematical, Engineering and Management Sciences

Vol. 4, No. 2, 361-374, 2019

https://dx.doi.org/10.33889/IJMEMS.2019.4.2-029

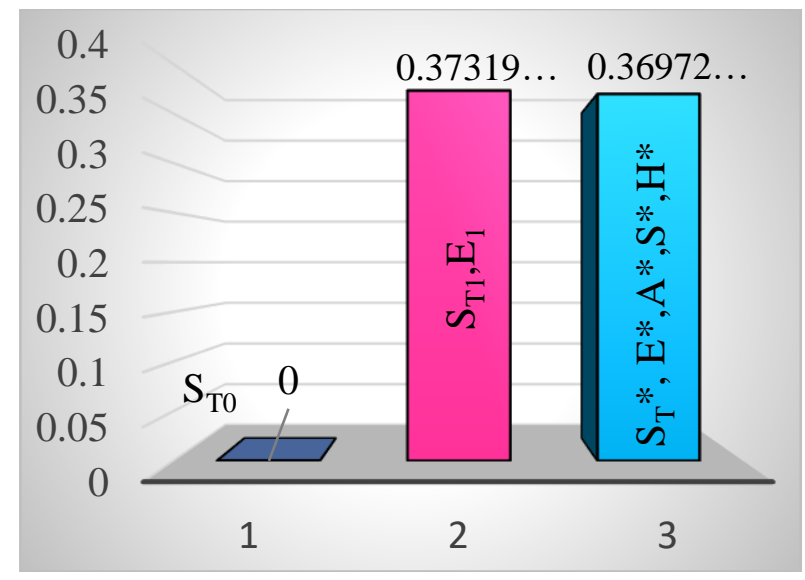

Figure 8. Graph representation of reproduction number

Figure 7 shows that $2 \%$ individuals are susceptible out of which $11 \%$ gets exposed. From that $11 \%$ exposed individuals, $37 \%$ are asymptomatic individuals and $28 \%$ are symptomatic out of which $22 \%$ gets hospitalized. Figure 8 shows that at every equilibrium point the value of reproduction number is less than 1 . Therefore, the mathematical model is stable for the given parametric values.

\section{Conclusion}

Using system of nonlinear ordinary differential equations, a mathematical model is formulated to study the hospitalization rate due to impact of temperature variations on human health. System is locally and globally asymptotically stable at all equilibrium points. A basic reproduction number has been evaluated at an equilibrium point and is equal to 0.3732 which shows that approx. $37 \%$ of the individuals in the society gets hospitalized due to temperature variations. To validate the model, numerical simulations has been carried out which interprets that with the increase in rate of exposed and asymptomatic compartments, increase of susceptible individuals are observed which further results in increased hospitalization rate and the mathematical model is stable at every equilibrium point. Hospitalization rate can be controlled by taking proper precautions at the early stages. As we know better health helps to face physical, mental and social challenges. So, it is essential to become hale and hearty human as it makes people live longer and more productive resulting in decrease in hospitalization rate. Hence, every individual in the society should take proper care of their body and respond immediately if symptoms are observed by taking proper medications.

\section{Conflict of Interest}

The authors confirm that there is no conflict of interest to declare for this publication.

\section{Acknowledgement}

The authors will like to thank reviewers for their positive and helpful comments. The authors thank DSTFIST file \# MSI-097 for technical support to the department. 
International Journal of Mathematical, Engineering and Management Sciences

Vol. 4, No. 2, 361-374, 2019

https://dx.doi.org/10.33889/IJMEMS.2019.4.2-029

\section{References}

Bandyopadhyay, S., Kanji, S., \& Wang, L. (2012). The impact of rainfall and temperature variation on diarrheal prevalence in Sub-Saharan Africa. Applied Geography, 33, 63-72.

Diekmann, O., Heesterbeek, J. A. P., \& Roberts, M. G. (2009). The construction of next-generation matrices for compartmental epidemic models. Journal of the Royal Society Interface, 7(47), 873-885.

Isea, R., \& Lonngren, K. E. (2016). A preliminary mathematical model for the dynamic transmission of dengue, chikungunya and zika. American Journal of Modern Physics and Application, 3(2), 11-15.

LaSalle, J. P. (1976). The Stability of Dynamical Systems, vol. 25 of Regional Conference Series in Applied Mathematics. Society for Industrial and Applied Mathematics.

McMichael, A. J. (2003). Global climate change and health: an old story writ large. Climate Change and Human Health: Risks and Responses. Geneva, Switzerland: World Health Organization.

McMichael, A. J., \& Haines, A. (1997). Global climate change: the potential effects on health. BMJ: British Medical Journal, 315(7111), 805.

Paaijmans, K. P., Blanford, S., Bell, A. S., Blanford, J. I., Read, A. F., \& Thomas, M. B. (2010). Influence of climate on malaria transmission depends on daily temperature variation. Proceedings of the National Academy of Sciences of the United States of America, 107(34), 15135-15139.

Qiu, H., Yu, I. T. S., Tse, L. A., Chan, E. Y., Wong, T. W., \& Tian, L. (2015). Greater temperature variation within a day associated with increased emergency hospital admissions for asthma. Science of the Total Environment, 505, 508-513.

Routh, E. J. (1877). A treatise on the stability of a given state of motion: particularly steady motion. London: Macmillan and Company.

Shah, N. H., \& Gupta, J. (2014). Mathematical modeling of Malaria using differential Equations., C. N. B. Herrera (Ed.), Deforestation: Conservation Policies, Economic Implications and Environmental Impact (pp. 115-132), New York: Nova Science Publishers, Inc., USA.

Shah, N. H., \& Gupta, J. (2013). SEIR model and simulation for vector borne diseases. Applied Mathematics, $4(8), 13$.

Shah, N. H., Patel, S. N., Satia, M. H., \& Thakkar, F. A. Optimal Control for Transmission of Water Pollutants. (2018) Optimal Control for Transmission of Water Pollutants. International Journal of Mathematical, Engineering and Management Sciences, 3(4), 381-391.

Shahgholian, K., \& Hajihosseini, H. (2009). A Dynamic Model of air pollution, health, and population growth using system dynamics: a study on Tehran-Iran (with computer simulation by the software Vensim). International Journal of Environment, Chemical Ecological, Geological and Geophysical Engineering, World Academy of Science, Engineering and Technology, 3(11), 372-379.

Wang, X. Y., Barnett, A. G., Hu, W., \& Tong, S. (2009). Temperature variation and emergency hospital admissions for stroke in Brisbane, Australia, 1996-2005. International journal of Biometeorology, 53(6), 535-541.

Wu, X., Lu, Y., Zhou, S., Chen, L., \& Xu, B. (2016). Impact of climate change on human infectious diseases: Empirical evidence and human adaptation. Environment International, 86, 14-23.

Yakob, L., \& Clements, A. C. (2013). A mathematical model of chikungunya dynamics and control: the major epidemic on Réunion Island. PloS one, 8(3), e57448.

Zell, R., Krumbholz, A., \& Wutzler, P. (2008). Impact of global warming on viral diseases: what is the evidence? Current Opinion in Biotechnology, 19(6), 652-660. 
International Journal of Mathematical, Engineering and Management Sciences

Vol. 4, No. 2, 361-374, 2019

https://dx.doi.org/10.33889/IJMEMS.2019.4.2-029

(c) (7) Original content of this work is copyright (c) International Journal of Mathematical, Engineering and Management Sciences. All rights reserved Except of uses under a Creative Commons Attribution 4.0 International (CC BY 4.0) license at https://creativecommons.org/licenses/by/4.0/ 